\title{
Role of Age-Associated Alterations of the Dermal Extracellular Matrix Microenvironment in Human Skin Aging: A Mini-Review
}

\author{
Taihao Quan Gary J. Fisher \\ Department of Dermatology, University of Michigan Medical School, Ann Arbor, Mich., USA
}

\begin{abstract}
Key Words
Skin · Aging · Cysteine-rich protein $61 \cdot$ Age-associated dermal microenvironment $\cdot$ Collagen · Transforming growth factor- $\beta$. Matrix metalloproteinases · Inflammaging
\end{abstract}

\begin{abstract}
Human skin is largely composed of a collagen-rich connective tissue, which provides structural and functional support. The collagen-rich connective tissue is produced, organized, and maintained by dermal fibroblasts. During aging, dermal collagen fibrils undergo progressive loss and fragmentation, leading to thin and structurally weakened skin. Age-related alterations of collagen fibrils impairs skin structure and function and creates a tissue microenvironment that promotes age-related skin diseases, such as delayed wound healing and skin cancer development. This mini-review describes cellular mechanisms that give rise to self-perpetuating, collagen fibril fragmentation that creates an age-associated dermal microenvironment, which contributes to decline of human skin function.

(c) 2015 S. Karger AG, Basel
\end{abstract}

\section{Introduction}

Human skin is the largest and most massive organ of the human body. The primary function of the skin is to provide a protective barrier from the environment, such as heat, solar ultraviolet (UV) irradiation, infection, injury, and water loss. Human skin consists of two layers: the epidermis (outermost layer) and the dermis (vascular connective tissue below the epidermis), which resides above subcutaneous adipose tissue. The epidermis is primarily composed of keratinocytes, which produce keratins, intermediate filaments that provide mechanical stability. Keratinocytes continually undergo terminal differentiation; a process that results in formation of the outermost protective barrier (horny layer or stratum corneum). The dermis is largely composed of dense collagen-rich extracellular matrix (ECM). Dermal collagen represents by far the most abundant ECM protein and constitutes the bulk of skin ( $90 \%$ dry weight) [1]. Dermal connective tissue collagen is essentially responsible for the skin's tensile strength and mechanical properties. In human skin dermis, collagen-rich ECM is synthesized, organized, and maintained by dermal fibroblasts. Therefore, impaired dermal fibroblast function has significant impact on the properties of skin connective tissue.

Human skin, like all other organs, undergoes a natural aging process as a consequence of the passage of time. In addition, human skin, unlike other organs, continuously experiences harmful stress and damage from environmental sources such as solar UV irradiation. Based on its causes, cutaneous aging is classified into two types: natural aging, also known as intrinsic aging, and photoaging, also known as extrinsic aging. Natural aging refers to

\section{KARGER 125}

(c) 2015 S. Karger AG, Base

0304-324X/15/0615-0427\$39.50/0

E-Mail karger@karger.com

www.karger.com/ger
Gary J. Fisher

Department of Dermatology, University of Michigan Medical School

6447 Med Sci I, 1301 E Catherine

Ann Arbor, MI 48109 (USA)

E-Mail gifisher@umich.edu 

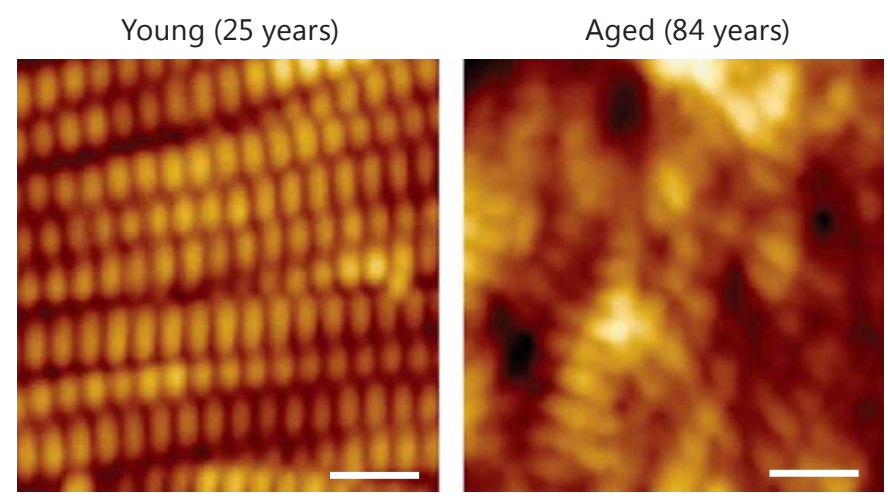

Fig. 1. Alterations of dermal connective tissue collagen are characteristic features of human skin aging. Nanoscale human skin dermal collagen fibrils were analyzed by AFM. Representative AFM images of dermal collagen fibrils in young (25 years) and aged (84 years) sun-protected underarm skin. Bars $=100 \mathrm{~nm}$.

those changes observed in all individuals resulting from the passage of time, whereas photoaging refers to those changes attributable to habitual sun exposure. Both processes are cumulative, and therefore, photoaging is superimposed on intrinsic aging. The most clinically noticeable age-related changes occur on face, neck, forearm, and lower leg skin [2]. These areas undergo a combination of natural aging and photoaging and age-related skin diseases occur most often in these areas.

The clinical signs of natural aging and photoaging skin are different [3]. Natural skin aging is characterized by laxity and fine wrinkling, but is not associated with increased pigmentation, deep wrinkles, or leathery appearance that are characteristic features of photoaging skin. There are histological differences between natural aging and photoaging skin as well [4]. In naturally aged skin, the epidermis is atrophic with flattening of the dermalepidermal junction and thin dermis. Photoaged skin, in contrast, is often associated with increased epidermal thickness and severe dermal connective tissue damage including accumulation of elastin-containing material, known as solar elastosis [5].

Histological and ultrastructural studies have revealed that the major alterations in aged skin are localized in the dermal ECM [6, 7]. Figure 1 shows nanoscale topographical images of dermal collagen fibrils assessed by atomic force microscopy (AFM). In young (20-30 years) skin, intact collagen fibrils are abundant, tightly packed, and well-organized. In contrast, in aged skin ( $>80$ years), collagen fibrils are fragmented and disorganized. Investigations have revealed that characteristic features of skin connective tissue aging are largely due to aberrant collagen homeostasis. Two interrelated mechanisms are involved; reduced collagen biosynthesis and increased collagen fibril fragmentation. These mechanisms result in net collagen deficiency, which manifests as thin, fragile skin $[7,8]$. Age-related aberrant collagen homeostasis impairs the structural integrity and mechanical properties of the skin, and contributes to age-related skin diseases.

All procedures involving human subjects were conducted in accordance with the regulations set forth by the University of Michigan Institutional Review Board, and all subjects provided written informed consent.

\section{Impaired Transforming Growth Factor- $\beta$ Signaling and Skin Connective Tissue Aging}

A wealth of evidence indicates that transforming growth factor- $\beta$ (TGF- $\beta$ ) plays a central role in ECM biosynthesis [9]. In human dermal fibroblasts, TGF- $\beta$ controls collagen homeostasis by regulation of both collagen synthesis and degradation [8]. TGF- $\beta$ initiates its cellular action by binding to its cell surface receptor complex, composed of TGF- $\beta$ type I receptor (T $\beta \mathrm{RI})$ and TGF- $\beta$ type II receptor (T $\beta$ RII). Binding of TGF- $\beta$ allows T $\beta$ RII to activate T $\beta R I$, which in turn activates the transcription factors Smad2 and Smad3. Activated Smad2 or Smad3 forms heteromeric complexes with the common partner Smad4. These activated Smad complexes translocate into the nucleus, where they interact with Smad binding elements in the promoter regions of TGF- $\beta$ target genes. Identification of TGF- $\beta /$ Smad target genes in dermal fibroblasts using a cDNA microarray revealed that 265 known ECM and ECM-related genes were regulated by TGF- $\beta /$ Smad signaling [10]. For example, many ECM genes, such as multiple collagens, fibronectin, decorin, and versican were directly upregulated by TGF- $\beta / \mathrm{Smad}$ signaling. In contrast, TGF- $\beta$ downregulates matrix-degrading metalloproteinases (MMPs) and upregulates tissue inhibitor of metalloproteases and plasminogen activator inhibitor 1 , which inhibit MMPs activation. These data suggest that the TGF- $\beta /$ Smad pathway is not only enhancing ECM gene expression, but also inhibiting ECM degradation.

As such, the TGF- $\beta /$ Smad pathway is recognized as a primary regulator of ECM homeostasis. Deregulation of TGF- $\beta$ signaling plays a prominent role in the pathogenesis associated with ECM proteins. For example, upregulation of TGF- $\beta$ signaling causes abnormal accumulation 
of ECM proteins in affected tissues, as seen in systemic sclerosis, a chronic and progressive connective tissue disease characterized by fibrosis of the skin and internal organs. In contrast, downregulation of TGF- $\beta$ signaling negatively regulates collagen homeostasis and has a significant impact on the aging of human skin connective tissue $[8,11]$. Laser capture microdissection coupled with quantitative real-time RT-PCR indicates that the T $\beta$ RII mRNA level is reduced by $59 \%$ in aged ( $>80$ years), compared to young (20-30 years) skin dermis [12]. In contrast to T $\beta R I I, T \beta R I m R N A$ levels are similar in aged and young human skin. Interestingly, reactive oxygen species (ROS), which are considered to play a pivotal role in the biology of aging, substantially reduce T $\beta$ RII, but not T $\beta R I$, gene expression $[11,13]$.

As described above, TGF- $\beta$ initiates its cellular actions by binding its cell surface receptor complex. Reduced expression of T $\beta$ RII causes decreased binding of TGF- $\beta$ to the surface of dermal fibroblasts and reduced T $\beta$ RII signal transduction [14]. Electrophoretic mobility shift assays, using the Smad3 binding element as probe, shows that loss of T $\beta$ RII substantially reduces formation of the TGF- $\beta$-induced DNA/Smad complexes [15]. Preventing loss of T $\beta$ RII by overexpression restores TGF- $\beta$ activation of Smad3, and therefore protects against ROS inhibition of type I procollagen gene expression $[11,13]$. Thus, downregulation of T $\beta$ RII appears to be a critical event in age-related decline of dermal collagen production, with attendant skin thinning, observed in aged human skin.

\section{Elevated MMPs and Skin Connective Tissue Aging}

MMPs, which cleave collagen and other ECM proteins, are elevated in naturally aged human skin $[16,17]$. Several MMPs are elevated in aged human skin [17]. These MMPs can be divided into the following groups: collagenases, MMP-1; gelatinases, MMP-2 and MMP-9; stromelysins, MMP-3 and MMP-11; membrane-associated, MMP-17, and the recently identified MMP-27. Furthermore, laser capture microdissection coupled with real-time RT-PCR indicates that these elevated MMPs are primarily produced by dermal fibroblasts in human skin in vivo. Additionally, elevation of MMPs in aged skin is not accompanied by alterations of endogenous MMP inhibitors (tissue inhibitor of metalloproteases) [17].

In human skin, MMP-1 is the major protease capable of initiating fragmentation of native fibrillar collagens, predominantly type I and III collagens. MMP-1 cleaves collagen fibrils at a single site within its central triple he-

Age-Associated Alterations of the Dermal

ECM Microenvironment in Skin Aging lix. Once cleaved by MMP-1, collagen can be further degraded by MMP-3 and MMP-9 $[18,19]$. Dermal collagen fibrils are stabilized by cross-links that are highly resistant to proteolytic cleavage. Therefore, the actions of elevated MMPs over decades are thought to result in accumulation of cross-linked collagen fragments, which impair the structure and function of the dermal ECM. Exposure of sun-protected human skin to purified human MMP-1 in human skin organ culture causes collagen fragmentation, and alterations in the structure and organization of collagen fibrils in the dermis that resemble those observed in aged skin [16]. The above data support the concept that the combined actions of multiple MMPs, which are constitutively elevated in aged dermis, are major mediators of skin connective tissue damage that is observed in aged human skin.

\section{Elevated Cysteine-Rich Protein 61 Promotes Age-Associated Dermal Microenvironment}

Emerging evidence indicates that elevated cysteinerich protein 61 (CCN1) [20], a member of the CCN (ㄷR61/TGF/NOV) family of proteins, mediates aberrant collagen homeostasis in dermal fibroblasts and contributes to human skin connective tissue aging [12, 2123 ]. The $\mathrm{CCN}$ family comprises six distinct members: CCN1, connective tissue growth factor (CCN2), nephroblastoma overexpressed (CCN3), Wnt-inducted secreted protein-1 (CCN4), Wnt-inducted secreted protein-2 (CCN5), and Wnt-inducted secreted protein-3 (CCN6) [24]. CCN proteins are secreted, associate with ECM proteins, and exhibit diverse functions involved, inflammation, cell-matrix interactions, regulation of ECM proteins synthesis, and wound healing, in a variety of cell types [25]. Studies in animal models and in patients have confirmed that $\mathrm{CCN}$ proteins are involved in many diseases related to inflammation and tissue repair [25].

In human skin, $\mathrm{CCN} 1$ is predominantly expressed in dermal fibroblasts $[12,22]$. Interestingly, CCN1 is markedly elevated in naturally aged and photoaged human skin in vivo $[12,26,27]$. In cultured human skin fibroblasts, elevated expression of CCN1 substantially reduces type I procollagen and concurrently increases MMP-1 $[12,17,22]$. In further investigations of CCN1 actions, we found that elevated CCN1 in human dermal fibroblasts alters expression of numerous secreted proteins, and that the patterns of CCN1-induced alterations closely resemble those observed in aged dermis [12, 17, 21, 27]. We refer collectively to CCN1-induced alterations of the 


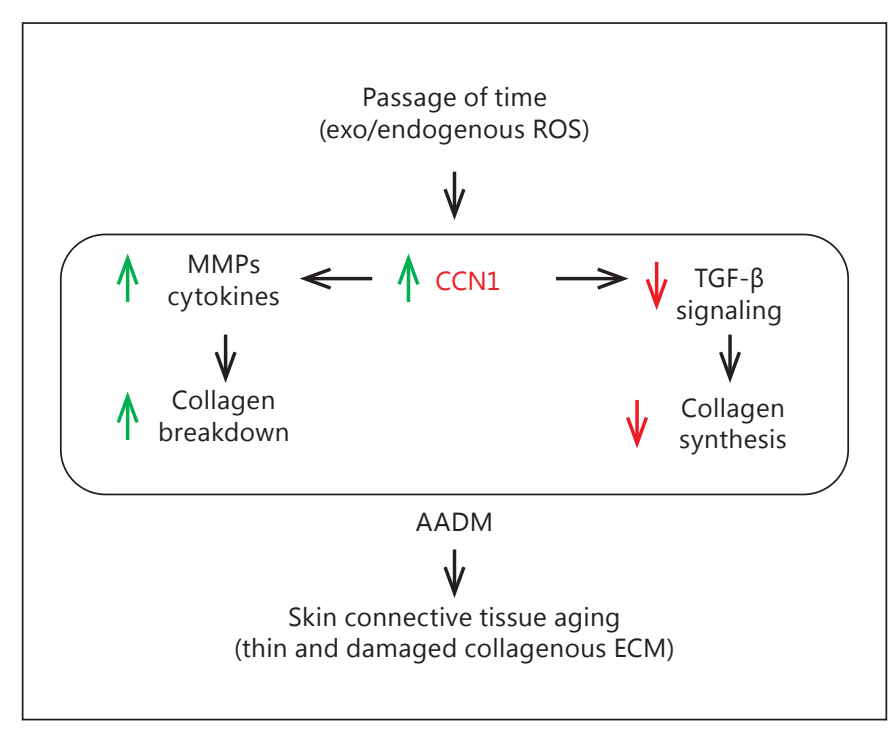

Fig. 2. Model for human skin connective tissue aging. Human skin is a primary target of ROS generated from both extrinsic and intrinsic sources, such as UV irradiation from the sun and metabolically generated pro-oxidants. The passage of time and chronic exposure to ROS increases the AP-1 complex, which in turn upregulates $\mathrm{CCN} 1$ expression. Elevated CCN1 alters dermal fibroblast function leading to development of AADM: (1) AADM encompasses reduced production of collagens (thin skin dermis); (2) increased production of multiple MMPs (collagen fibril fragmentation), and (3) creation of a proinflammatory microenvironment (inflammaging), and thus AADM promotes skin connective tissue aging. AADM impairs dermal structural integrity and mechanical properties and creates a tissue microenvironment that contributes to age-related decline of skin function, such as reduced strength and delayed wound healing, and promotes epithelial skin cancer.

dermis as age-associated dermal microenvironment (AADM) (fig. 2).

CCN1-induced AADM promotes skin connective tissue aging through three major mechanisms: (1) reduced production of dermal ECM components, such as type I and type III collagens, which contributes to dermal thinning; (2) induction of multiple MMPs (MMP-1, MMP-3, MMP-9, MMP-10, and MMP-23), which promote fragmentation of ECM proteins, and (3) increased expression of proinflammatory cytokines (IL-1 $\beta$, IL-6, and IL-8), which promotes inflammatory microenvironment (inflammaging). CCN1-induced AADM accounts for many of the characteristic features of aged human skin dermis, including loss of tissue and decline of function.

Figure 2 depicts a model in which elevated CCN1 in aged dermal fibroblasts contributes to human skin aging through creating AADM. Like other organs, human skin is exposed to ROS generated from aerobic metabolism. In addition, human skin is a major target for a broad spectrum of external stressors, such as solar UV radiation as well as microbial and chemical assaults. Chronic exposure to ROS upregulates CCN1 expression. Elevated CCN1 impairs dermal fibroblast production of collagen by inhibiting TGF- $\beta$ signaling, and promoting production of MMPs and proinflammatory cytokines. These alterations lead to thin and fragmented dermal collagenous ECM, characteristic features of aged human skin. Elevated expression of CCN1 in human dermal fibroblasts acts through multiple pathways to promote AADM: (1) impairment of TGF- $\beta$ signaling by downregulation of T $\beta$ RII, and thus contributes to age-associated thinning of the dermis [12]; (2) induction of multiple MMPs via upregulation of transcription factor AP-1, a major regulator of multiple MMPs, and thus contributes to age-associated ECM fragmentation [12, 21, 22], and (3) elevation of multiple proinflammatory cytokines, and thus contributes to age-associated inflammatory microenvironment (inflammaging) $[21,23]$.

CCN1-induced proinflammatory cytokines may have a significant impact on the development of AADM. For example, IL-1 $\beta$, an AADM-associated cytokine, not only upregulates multiple MMPs but also downregulates type I collagen synthesis [23]. IL-1 $\beta$ is elevated in the dermis of naturally aged and photoaged human skin [23]. CCN1 markedly induces IL-1 $\beta$, which in turn contributes to CCN1-mediated reduction of type I collagen expression and induction of MMP-1 expression.

Emerging evidence indicates that the CCN family proteins may represent a new class of modulators of inflammation [28]. Consistent with this notion, CCN1 activates a proinflammatory genetic program in murine macrophages [29]. Furthermore, evidence indicates a potential role of CCN1 in chronic inflammatory diseases such as atherosclerosis, rheumatoid arthritis, inflammatory kidney diseases and neuroinflammatory diseases [25]. There is a strong association of aging with chronic low-grade inflammatory activity which may progress to long-term tissue damage and systemic chronic inflammation [30]. Accumulating evidence supports the concept of 'inflammaging', which posits that low-grade chronic elevation of proinflammatory mediators can be a driving force for the aging progress [31]. Central to this concept is that healthy aging is not an inflammatory disease, but rather that subclinical inflammation contributes to the gradual decline of organ function, which occurs during the aging process. For example, IL-6 is increased in the aged and has been suggested to be a 
marker of health status in the elderly [32]. Interestingly, IL-6 is markedly induced by CCN1 in human skin dermal fibroblasts and constitutively elevated in aged skin [26]. Although aged skin does not display overt inflammation, the possibility that AADM-associated cytokines contribute to human skin connective tissue aging deserves further investigation.

It has been shown that $\mathrm{CCN}$ proteins function primarily through interactions with integrins in a cell-type, function-specific manner [33]. CCN1 has been reported to interact with integrins to increase intracellular levels of ROS, which function as important effectors of CCN1 actions $[28,34]$. The ability of CCN1 to induce AADM in dermal fibroblasts may be mediated by integrin-coupled generation of ROS. It is well-documented that the integrin pathway and ROS lead to activation of transcription factor AP-1, which upregulates MMPs and proinflammatory cytokines, including IL- $1 \beta$ and IL- $6[23,28]$. Therefore, it is conceivable that elevated CCN1 upregulates MMPs and cytokines through activation of integrin and/ or ROS-mediated activation of AP-1 in aged human skin dermal fibroblasts. Data indicate that CCN1 is not only induced by oxidative stress, but also increases intracellular levels of ROS, suggesting that a positive feedback loop involving CCN1 and ROS/AP-1 may result in sustained elevation of ROS and CCN1 in fibroblasts in aged human skin.

Studies in a mouse model have demonstrated that CCN1 exerts potent antifibrotic activity via induction of myofibroblast senescence during cutaneous wound healing [28]. As replicative senescence is a form of cellular aging, $\mathrm{CCN} 1 \mathrm{mRNA}$ and protein levels are significantly elevated in replicative senescent dermal fibroblasts [35]. Replicative senescent dermal fibroblasts also express significantly reduced levels of type I procollagen and increased levels of MMP-1, as observed in aged human skin. Knockdown of elevated CCN1 in senescent dermal fibroblasts partially normalizes both type I procollagen and MMP-1 expression. These data further support a key role of CCN1 in regulation of collagen homeostasis, and elevated $\mathrm{CCN} 1$ functions as an important mediator of age-related reduction of type collagen in aged human skin.

Vitamin A and its metabolites have been shown to improve naturally aged and photoaged skin by promoting deposition of new collagen and preventing its degradation [36]. In skin equivalent cultures, all-trans retinoic acid, the major bioactive form of vitamin A in skin, significantly increases type I procollagen, and reduces MMP1 [35]. In contrast, addition of recombinant human CCN1 to skin equivalent cultures significantly reduces type I procollagen and increases MMP-1 [35]. Interestingly, alltrans retinoic acid significantly reduces $\mathrm{CCN} 1$ expression in skin equivalent cultures [35]. Topical treatment with retinol (vitamin A, $0.4 \%$ ) for 7 days significantly reduces CCN1 gene expression, compared to vehicle-treated skin, in both naturally aged and photoaged human skin in vivo [35]. These data suggest that the mechanism by which retinoids improve aged skin, through increased collagen production, may involve downregulation CCN1.

\section{AADM and Age-Related Skin Diseases}

Recent studies have indicated that alterations in the stromal microenvironment, like AADM, play a significant role in age-related diseases, for example, impaired wound healing and greater risk of cancer development $[37,38]$. Epithelial skin cancer is the most common type of cancer in the United States, with over two million cases diagnosed annually [39]. An intriguing question is why cancers occur more frequently in elderly, compared to young populations. For example, $40-50 \%$ of Americans who live to age 65 years will have skin cancer at least once $[39,40]$. Development of skin cancer is associated with chronic exposure to solar UV radiation and age. It is possible that alterations in the dermis caused by UV irradiation may create a microenvironment that fosters development of epithelial skin cancer. Indeed, it has been suggested that alterations in dermal ECM/stroma that occur during the skin aging process provide a microenvironment that supports the development of skin cancers [41].

Emerging evidence indicates that young tissue microenvironment (healthy architecture and normal tissue homeostasis) provides tumor-suppressive signals [42]. However, as tissue homeostasis becomes disrupted with aging, for example due to emergence of AADM in elderly, the tissue microenvironment can promote tumor formation [43]. Recent studies have demonstrated that alterations in the stromal microenvironment, such as disruption of Notch [44] or TGF- $\beta$ signaling [45] in dermal fibroblast, can induce epithelial tumorigenesis. Furthermore, elevated Wnt (Wingless-type MMTV integration site family) [46] and HGF (hepatocyte growth factor) [41] signaling in stromal fibroblasts can promote cancer drug resistance and control drug sensitivity. Although the influence of the stromal microenvironment on tumor progression is well recognized, little is known about the role of age-related aberrant dermal microenvironment, like AADM, in skin cancer development. It is conceivable 


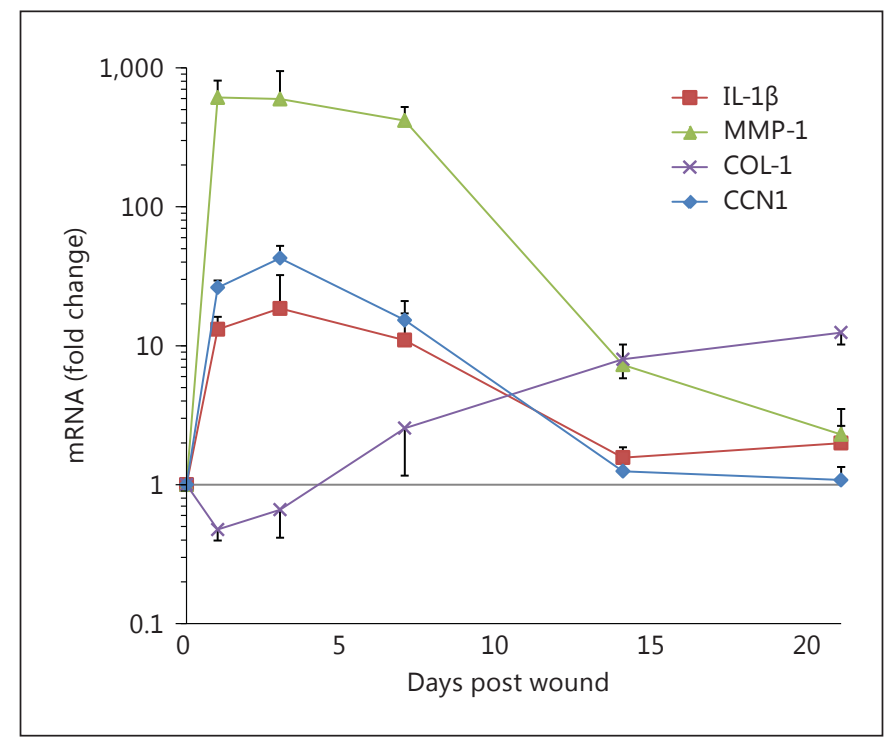

Fig. 3. Wounding rapidly induces $\mathrm{CCN} 1$ in human skin in vivo. Partial thickness wounds were made in sun-protected buttock skin of healthy adult individuals by $\mathrm{CO}_{2}$ laser. Skin samples were obtained at indicated times, and mRNA levels were quantified by real-time RT-PCR and normalized to mRNA internal control housekeeping gene 36B4. Values are means $\pm \operatorname{SEM}(n=6)$.

that CCN1-induced AADM contributes to epithelial skin cancer development in the aged.

CCN1-induced AADM may also have a significant impact on delayed wound healing in elderly. We have found that $\mathrm{CO}_{2}$ laser ablation, which causes partial thickness wound, rapidly and markedly induces $\mathrm{CCN} 1$ in human skin dermis in vivo (fig. 3). CCN1 is induced within 1 day (22-fold), reaches the maximum at 3 days ( 31 -fold), and returns to the basal level at 3 weeks postwounding. It is important to note that the timing of elevated CCN1 levels corresponds to the inflammatory phase (ECM breakdown) and the timing of CCN1 diminution corresponds to the ECM remodeling phase (collagen production) of the wound healing response in human skin in vivo. Also of note is that CCN1-regulated AADM factors such as proinflammatory cytokine IL- $1 \beta$, collagen-degrading MMPs (MMP-1, MMP-3, and MMP-9), and type I collagen are key components of the wound healing response.

These data suggest that $\mathrm{CCN} 1$ may play a critical role in regulating wound repair in human skin. Further support comes from mouse studies, which have reported that CCN1 is induced by cutaneous wounding and participates in both the inflammatory and remodeling phases of repair [47]. These findings suggest that constitutive elevation CCN1 in aged human skin may delay wound healing process by exaggerated/extended inflammatory phase and delayed/defective remodeling phase.

\section{Future Directions}

This mini-review emphasizes the importance of alterations of the protein composition and organization of the dermal ECM as a driving force for human skin aging. The term 'core matrisome' has recently been coined to describe the group of collagens, proteoglycans, and glycoproteins that comprise the ECM in mammalian tissues [48]. This group of proteins contains 43 collagen subunits, 35 proteoglycans, and 200 glycoproteins. CCN1, as well as all other members of the CCN family, is included in the glycoproteins. Proteoglycans, a family of glycosaminoglycan (GAG)-conjugated proteins, are important constituents of human skin connective tissue and are essential for maintaining dermal homeostasis. We reported that quantitative gene expression of 20 known interstitial proteoglycans in human skin revealed that decorin was the most highly expressed followed by biglycan and versican [49]. Interestingly, the amount of total sulfated GAGs was significantly reduced by approximately $40 \%$ in aged skin compared to young skin, suggesting that agerelated alteration of GAGs may also contribute to skin aging. In addition to the core matrisome, the human matrisome includes core matrisome-associated proteins, such as ECM-bound secreted factors (e.g. TGF- $\beta$ and cytokines) and ECM regulators (e.g. MMPs and ADAMs).

Therefore, examples presented above of age-related alterations of specific dermal ECM proteins, and their roles in human skin aging, can be viewed in the context of the matrisome. Comprehensive understanding of age-related alterations of the composition of the human dermal matrisome, and mechanisms that bring about these alterations, will likely yield important novel insights into the molecular basis of skin aging and age-related skin diseases.

\section{Acknowledgements}

Some of the studies described in this article were supported by the National Institutes of Health (Bethesda, Md., USA) Grants: ES014697 and ES014697 30S1 to T.Q., AG019364 to G.J.F. and T.Q., and AG031452 and AG025186 to G.J.F. 


\section{References}

1 Uitto J: Connective tissue biochemistry of the 15 Quan T, He T, Kang S, Voorhees JJ, Fisher GJ: aging dermis. Age-related alterations in collagen and elastin. Dermatol Clin 1986;4:433446.

2 Wlaschek M, Tantcheva-Poor I, Naderi L, Ma W, Schneider LA, Razi-Wolf Z, Schuller J, Scharffetter-Kochanek K: Solar UV irradiation and dermal photoaging. J Photochem Photobiol B 2001;63:41-51.

-3 Yaar M, Eller MS, Gilchrest BA: Fifty years of skin aging. J Investig Dermatol Symp Proc 2002;7:51-58.

4 Kurban RS, Bhawan J: Histologic changes in skin associated with aging. J Dermatol Surg Oncol 1990;16:908-914.

5 Lavker R: Cutaneous aging: chronologic versus photoaging; in Gilchrest B (ed): Photodamage. Cambridge, Blackwell, 1995, vol 1, pp 123-135.

6 Fisher GJ, Wang ZQ, Datta SC, Varani J, Kang S, Voorhees JJ: Pathophysiology of premature skin aging induced by ultraviolet light. $\mathrm{N}$ Engl J Med 1997;337:1419-1428.

7 Fisher GJ, Varani J, Voorhees JJ: Looking older: fibroblast collapse and therapeutic implications. Arch Dermatol 2008;144:666-672.

>8 Quan T, Shao Y, He T, Voorhees JJ, Fisher GJ: Reduced expression of connective tissue growth factor (CTGF/CCN2) mediates collagen loss in chronologically aged human skin. J Invest Dermatol 2010;130:415-424.

$>9$ Varga J, Rosenbloom J, Jimenez SA: Transforming growth factor beta (TGF beta) causes a persistent increase in steady-state amounts of type I and type III collagen and fibronectin mRNAs in normal human dermal fibroblasts. Biochem J 1987;247:597-604.

-10 Verrecchia F, Chu ML, Mauviel A: Identification of novel TGF-beta/Smad gene targets in dermal fibroblasts using a combined cDNA microarray/promoter transactivation approach. J Biol Chem 2001;276:17058-17062.

-11 Quan T, He T, Kang S, Voorhees JJ, Fisher GJ: Solar ultraviolet irradiation reduces collagen in photoaged human skin by blocking transforming growth factor-beta type II receptor/ Smad signaling. Am J Pathol 2004;165:741751.

-12 Quan T, He T, Shao Y, Lin L, Kang S, Voorhees JJ, Fisher GJ: Elevated cysteine-rich 61 mediates aberrant collagen homeostasis in chronologically aged and photoaged human skin. Am J Pathol 2006;169:482-490.

$>13$ He T, Quan T, Shao Y, Voorhees JJ, Fisher GJ: Oxidative exposure impairs TGF-beta pathway via reduction of type II receptor and Smad3 in human skin fibroblasts. Age (Dordr) 2014;36:9623.

14 Quan T, He T, Voorhees JJ, Fisher GJ: Ultraviolet irradiation blocks cellular responses to transforming growth factor-beta by downregulating its type-II receptor and inducing Smad7. J Biol Chem 2001;276:26349-26356. Ultraviolet irradiation alters transforming growth factor beta/Smad pathway in human skin in vivo. J Invest Dermatol 2002;119:499506.

16 Fisher GJ, Quan T, Purohit T, Shao Y, Cho MK, He T, Varani J, Kang S, Voorhees JJ: Collagen fragmentation promotes oxidative stress and elevates matrix metalloproteinase-1 in fibroblasts in aged human skin. Am J Pathol 2009;174:101-114.

17 Quan T, Little E, Quan H, Qin Z, Voorhees JJ, Fisher GJ: Elevated matrix metalloproteinases and collagen fragmentation in photodamaged human skin: impact of altered extracellular matrix microenvironment on dermal fibroblast function. J Invest Dermatol 2013;133: 1362-1366

18 Fisher GJ, Datta SC, Talwar HS, Wang ZQ, Varani J, Kang S, Voorhees JJ: Molecular basis of sun-induced premature skin ageing and retinoid antagonism. Nature 1996;379:335339.

19 Brennan M, Bhatti H, Nerusu K, BhagavathMatrix metalloproteinase-1 is the major collagenolytic enzyme responsible for collagen damage in UV-irradiated human skin. Photochem Photobiol 2003;78:43-48.

20 Brigstock DR, Goldschmeding R, Katsube KI, Lam SC, Lau LF, Lyons K, Naus C, Perbal B, Riser B, Takigawa M, Yeger H: Proposal for a unified CCN nomenclature. Mol Pathol 2003; 56:127-128.

21 Quan T, Qin Z, Robichaud P, Voorhees JJ, Fisher GJ: CCN1 contributes to skin connective tissue aging by inducing age-associated secretory phenotype in human skin dermal fibroblasts. J Cell Commun Signal 2011;5: 201-207.

22 Qin Z, Fisher GJ, Quan T: Cysteine-rich protein 61 (CCN1) domain-specific stimulation of matrix metalloproteinase-1 expression through $\alpha \mathrm{V} \beta 3$ integrin in human skin fibroblasts. J Biol Chem 2013;288:12386-12394.

23 Qin Z, Okubo T, Voorhees JJ, Fisher GJ, Quan T: Elevated cysteine-rich protein 61 (CCN1) promotes skin aging via upregulation of IL- $1 \beta$ in chronically sun-exposed human skin. Age 2014;36:353-364.

24 Perbal B: CCN proteins: multifunctional signalling regulators. Lancet 2004;363:62-64.

25 Jun JI, Lau LF: Taking aim at the extracellular matrix: CCN proteins as emerging therapeutic targets. Nat Rev Drug Discov 2011;10:945963.

26 Quan T, Shin S, Qin Z, Fisher GJ: Expression of CCN family of genes in human skin in vivo and alterations by solar-simulated ultraviolet irradiation. J Cell Commun Signal 2009;3:1923. ula N, Kang S, Fisher G, Varani J, Voorhees J:
27 Quan T, Qin Z, Xu Y, He T, Kang S, Voorhees JJ, Fisher GJ: Ultraviolet irradiation induces CYR61/CCN1, a mediator of collagen homeostasis, through activation of transcription factor AP-1 in human skin fibroblasts. J Invest Dermatol 2010;130:1697-1706.

28 Jun JI, Lau LF: The matricellular protein CCN1 induces fibroblast senescence and restricts fibrosis in cutaneous wound healing. Nat Cell Biol 2010;12:676-685.

-29 Bai T, Chen CC, Lau LF: Matricellular protein CCN1 activates a proinflammatory genetic program in murine macrophages. J Immunol 2010;184:3223-3232.

30 Daynes RA, Araneo BA, Ershler WB, Maloney C, Li GZ, Ryu SY: Altered regulation of IL-6 production with normal aging. Possible linkage to the age-associated decline in dehydroepiandrosterone and its sulfated derivative. J Immunol 1993;150:5219-5230.

31 Franceschi C, Capri M, Monti D, Giunta S, Olivieri F, Sevini F, Panourgia MP, Invidia L, Celani L, Scurti M, Cevenini E, Castellani GC, Salvioli S: Inflammaging and anti-inflammaging: a systemic perspective on aging and longevity emerged from studies in humans. Mech Ageing Dev 2007;128:92-105.

32 Maggio M, Guralnik JM, Longo DL, Ferrucci L: Interleukin- 6 in aging and chronic disease: a magnificent pathway. J Gerontol A Biol Sci Med Sci 2006;61:575-584

33 Chen CC, Lau LF: Functions and mechanisms of action of CCN matricellular proteins. Int J Biochem Cell Biol 2009;41:771-783.

34 Lau LF: CCN1/CYR61: The very model of a modern matricellular protein. Cell Mol Life Sci 2011;68:3149-3163.

35 Quan T, Qin Z, Voorhees JJ, Fisher GJ: Cysteine-rich protein 61 (CCN1) mediates replicative senescence-associated aberrant collagen homeostasis in human skin fibroblasts. J Cell Biochem 2012;113:3011-3018.

36 Kafi R, Kwak HS, Schumacher WE, Cho S, Hanft VN, Hamilton TA, King AL, Neal JD, Varani J, Fisher GJ, Voorhees JJ, Kang S: Improvement of naturally aged skin with vitamin A (retinol). Arch Dermatol 2007;143: 606-612.

37 Kudravi SA, Reed MJ: Aging, cancer, and wound healing. In Vivo 2000;14:83-92.

38 Bissell MJ, Kenny PA, Radisky DC: Microenvironmental regulators of tissue structure and function also regulate tumor induction and progression: the role of extracellular matrix and its degrading enzymes. Cold Spring Harb Symp Quant Biol 2005;70:343-356.

39 US: Cancer trends progress report 2011/2012 update. Sun Protection 2011/2012; http:// progressreport.cancer.gov/doc_detail.asp?pi $\mathrm{d}=1$ \&did=2007\&chid=71\&coid=711\&mid

40 Gloster HM Jr, Brodland DG: The epidemiology of skin cancer. Dermatol Surg 1996;22: 217-226. 
41 Straussman R, Morikawa T, Shee K, BarzilyRokni M, Qian ZR, Du J, Davis A, Mongare MM, Gould J, Frederick DT, Cooper ZA, Chapman PB, Solit DB, Ribas A, Lo RS, Flaherty KT, Ogino S, Wargo JA, Golub TR: Tumour micro-environment elicits innate resistance to RAF inhibitors through HGF secretion. Nature 2012;487:500-504.

42 Campisi J: Aging and cancer cell biology, 2008. Aging Cell 2008;7:281-284.

43 Sprenger CC, Plymate SR, Reed MJ: Agingrelated alterations in the extracellular matrix modulate the microenvironment and influence tumor progression. Int J Cancer 2010; 127:2739-2748.
$44 \mathrm{Hu}$ B, Castillo E, Harewood L, Ostano P, Reymond A, Dummer R, Raffoul W, Hoetzenecker W, Hofbauer GF, Dotto GP: Multifocal epithelial tumors and field cancerization from loss of mesenchymal CSL signaling. Cell 2012; 149:1207-1220.

45 Bhowmick NA, Chytil A, Plieth D, Gorska AE, Dumont N, Shappell S, Washington MK, Neilson EG, Moses HL: TGF- $\beta$ signaling in fibroblasts modulates the oncogenic potential of adjacent epithelia. Science 2004;303:848851.

46 Sun Y, Campisi J, Higano C, Beer TM, Porter P, Coleman I, True L, Nelson PS: Treatmentinduced damage to the tumor microenvironment promotes prostate cancer therapy resistance through WNT16B. Nat Med 2012;18: 1359-1368.
47 Chen CC, Mo FE, Lau LF: The angiogenic factor CYR61 activates a genetic program for wound healing in human skin fibroblasts. J Biol Chem 2001;276:47329-47337.

48 Hynes RO, Naba A: Overview of the matrisome - an inventory of extracellular matrix constituents and functions. Cold Spring Harb Perspect Biol 2012;4:a004903.

49 Li Y, Liu Y, Xia W, Lei D, Voorhees JJ, Fisher GJ: Age-dependent alterations of decorin glycosaminoglycans in human skin. Sci Rep 2013;3:2422. 